\title{
Corrigendum to "Charged Particle, Photon Multiplicity, and Transverse Energy Production in High-Energy Heavy-Ion Collisions"
}

\author{
Raghunath Sahoo, ${ }^{1}$ Aditya Nath Mishra, ${ }^{1}$ Nirbhay K. Behera, ${ }^{2}$ and Basanta K. Nandi ${ }^{2}$ \\ ${ }^{1}$ Indian Institute of Technology Indore, Indore 452017, India \\ ${ }^{2}$ Indian Institute of Technology Bombay, Mumbai 400067, India \\ Correspondence should be addressed to Raghunath Sahoo; raghunath.sahoo@cern.ch
}

Received 8 November 2016; Accepted 24 October 2017; Published 28 December 2017

Copyright (c) 2017 Raghunath Sahoo et al. This is an open access article distributed under the Creative Commons Attribution License, which permits unrestricted use, distribution, and reproduction in any medium, provided the original work is properly cited. The publication of this article was funded by SCOAP . $^{3}$

In the review article entitled "Charged Particle, Photon Multiplicity, and Transverse Energy Production in High-Energy Heavy-Ion Collisions" [1], the following two modifications are suggested to take care of some minor errors.

(1) There were some descriptive errors in last paragraph of Section 3.4 as well as in corresponding Figures 14, 15, and 16. The following modifications are done both in the text and in Figures 14, 15, and 16. In addition to that a new figure is also added to the literature as Figure 45.

The pseudorapidity distributions of charged particles in $\mathrm{Cu}+\mathrm{Cu}, \mathrm{Au}+\mathrm{Au}$, and $\mathrm{Pb}+\mathrm{Pb}$ most central collisions at different energies are shown in Figures 14, 15, and 16, respectively. These distributions are fitted with the double Gaussian function as given in equation (6) of "Charged Particle, Photon Multiplicity, and Transverse Energy Production in High-Energy Heavy-Ion Collisions," which are represented by solid lines. The widths $\left(\sigma_{1}\right.$ and $\left.\sigma_{2}\right)$ and the amplitude parameters $\left(A_{1}\right.$ and $\left.A_{2}\right)$ obtained from the double Gaussian fitting to $0-6 \%$ centrality RHIC data are already given in Tables 2, 3, and 4 . Now to explain the charged particle density distributions in terms of Landau hydrodynamics the pseudorapidity distribution of charged particles $\left(d N_{\mathrm{ch}} / d \eta\right)$ should be converted into rapidity distribution $\left(d N_{\mathrm{ch}} / d y\right)$ by applying a Jacobian transformation. For this one needs to know the information about particle ratio and spectra measurements in the midrapidity as described in [2]. After the Jacobian transformation to $d N_{\mathrm{ch}} / d \eta$ distribution of $\mathrm{Pb}+\mathrm{Pb}$ collisions at $\sqrt{s_{\mathrm{NN}}}=2.76 \mathrm{TeV}$, the rapidity distribution of charged particles is obtained, as shown in Figure 45 . Now the $d N_{\mathrm{ch}} / d y$ data is fitted with a Gaussian and Landau-Carruthers functions represented by solid and dotted lines, respectively. It can be seen from Figure 45 that the Gaussian function describes the data nicely. Similar exercises can be carried out to get the $d N_{\text {ch }} / d y$ from the $d N_{\mathrm{ch}} / d \eta$ after Jacobian transformation. Then the widths are obtained after fitting the $d N_{\mathrm{ch}} / d y$ distributions by Gaussian and Landau-Carruthers functions, respectively. The ratios of the widths obtained from the Gaussian $\left(\sigma_{\text {data }}\right)$ and LandauCarruthers $\left(\sigma_{\text {carrut. }}\right)$ fittings to $d N_{\mathrm{ch}} / d y$ are calculated for all the energies, which are shown in Figure 17 of "Charged Particle, Photon Multiplicity, and Transverse Energy Production in High-Energy Heavy-Ion Collisions." It can be seen from Figure 17 that the pion data at AGS and SPS energies are in close agreement with the Landau hydrodynamics, whereas the deviations increase at RHIC and LHC energies.

(2) Table 6 should replace Table 6 of Section 9 in "Charged Particle, Photon Multiplicity, and Transverse Energy Production in High-Energy Heavy-Ion Collisions." 
TABLE 6: $E_{T} / N_{\mathrm{ch}}\left(\mathrm{in} \mathrm{GeV}\right.$ ) for different center of mass energies as a function of $N_{\text {part }}$, the measure of collision centrality (shown in Figure 44). Different columns correspond to centrality classes in \%.

\begin{tabular}{|c|c|c|c|c|c|c|c|c|c|c|c|c|c|c|c|}
\hline$\sqrt{s_{\mathrm{NN}}}(\mathrm{GeV})$ & $0-5$ & $5-10$ & $10-15$ & $15-20$ & $20-25$ & $25-30$ & $30-35$ & $35-40$ & $40-45$ & $45-50$ & $50-55$ & $55-60$ & $60-65$ & $65-70$ & Experiment \\
\hline 7.7 & 0.86 & 0.89 & 0.89 & 0.88 & 0.88 & 0.88 & 0.88 & 0.88 & 0.89 & 0.89 & & & & & PHENIX \\
\hline 19.6 & 0.74 & 0.73 & 0.73 & 0.72 & 0.71 & 0.71 & 0.70 & 0.70 & 0.70 & 0.69 & & & & & PHENIX \\
\hline 27 & 0.79 & 0.79 & 0.79 & 0.79 & 0.79 & 0.80 & 0.79 & 0.80 & 0.80 & 0.79 & & & & & PHENIX \\
\hline 39 & 0.80 & 0.81 & 0.82 & 0.83 & 0.83 & 0.84 & 0.84 & 0.84 & 0.85 & 0.81 & & & & & PHENIX \\
\hline 130 & 0.87 & 0.87 & 0.87 & 0.87 & 0.88 & 0.87 & 0.88 & 0.88 & 0.88 & 0.87 & 0.86 & 0.85 & 0.86 & 0.84 & PHENIX \\
\hline 200 & 0.88 & 0.88 & 0.88 & 0.88 & 0.88 & 0.88 & 0.88 & 0.87 & 0.87 & 0.86 & 0.85 & 0.84 & 0.83 & 0.82 & PHENIX \\
\hline$\sqrt{s_{\mathrm{NN}}}(\mathrm{GeV})$ & $0-5$ & $5-10$ & $10-20$ & $20-30$ & $30-40$ & $40-50$ & $50-60$ & $60-70$ & $70-80$ & & & & & & \\
\hline 200 & 0.86 & 0.86 & 0.86 & 0.86 & 0.85 & 0.82 & 0.79 & 0.75 & 0.69 & & & & & & STAR \\
\hline$\sqrt{\sqrt{s_{\mathrm{NN}}}}(\mathrm{TeV})$ & $0-2.5$ & $2.5-5$ & $5-7.5$ & $7.5-10$ & $10-20$ & $20-30$ & $30-40$ & $40-50$ & $50-60$ & $60-70$ & $70-80$ & & & & \\
\hline 2.76 & 1.26 & 1.25 & 1.24 & 1.23 & 1.22 & 1.21 & 1.19 & 1.17 & 1.16 & 1.15 & 1.05 & & & & CMS \\
\hline
\end{tabular}

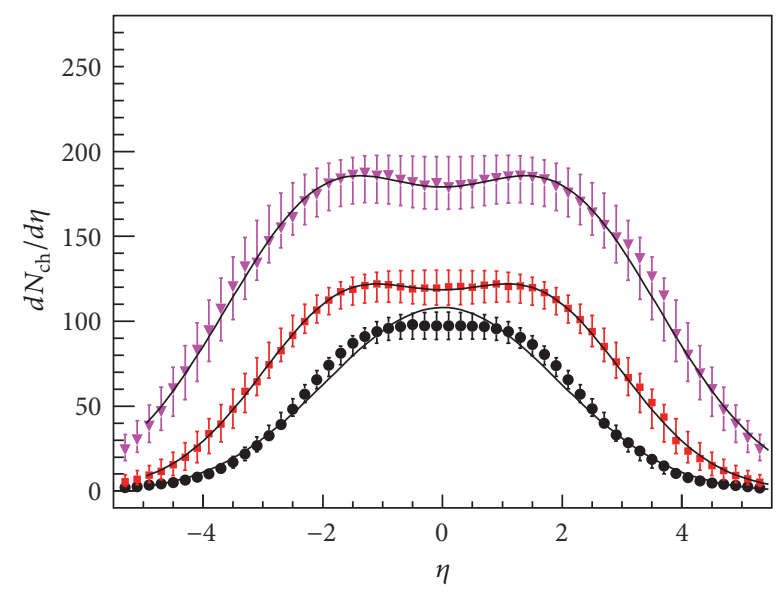

$$
\begin{aligned}
& \text { PHOBOS } \mathrm{Cu}+\mathrm{Cu}(0-6 \%) \quad-\text { Double Gaussian } \\
& +200 \mathrm{GeV} \\
& +62.4 \mathrm{GeV} \\
& +22.4 \mathrm{GeV}
\end{aligned}
$$

FIGURE 14: The pseudorapidity distribution of charged particles in $\mathrm{Cu}+\mathrm{Cu}$ collisions at three different energies, fitted with double Gaussian function represented by solid lines.

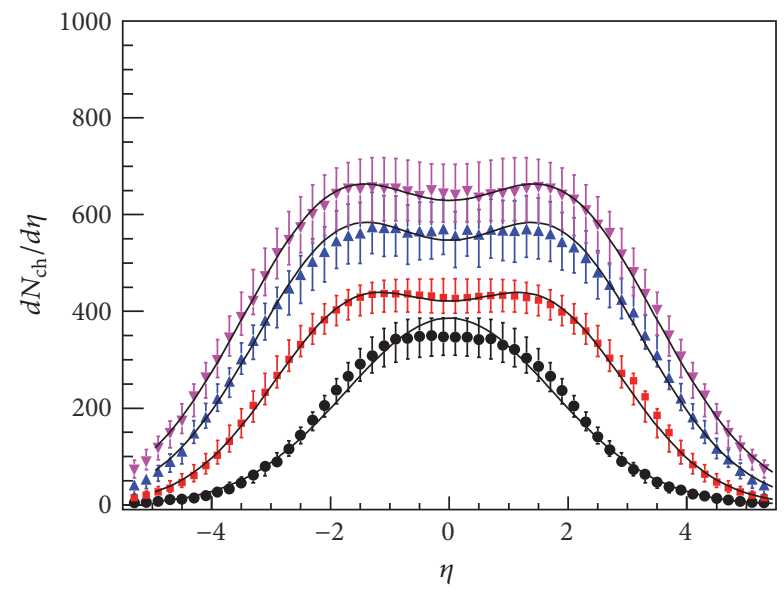

$$
\begin{aligned}
& \text { PHOBOS } \mathrm{Au}+\mathrm{Au}(0-6 \%)-\text { Double Gaussian } \\
& +200 \mathrm{GeV}+19.6 \mathrm{GeV} \\
& \text { * } 130 \mathrm{GeV} \\
& +62.4 \mathrm{GeV}
\end{aligned}
$$

FIGURE 15: The pseudorapidity distribution of charged particles in $\mathrm{Au}+\mathrm{Au}$ collisions at four different energies, fitted with double Gaussian function shown by solid lines.

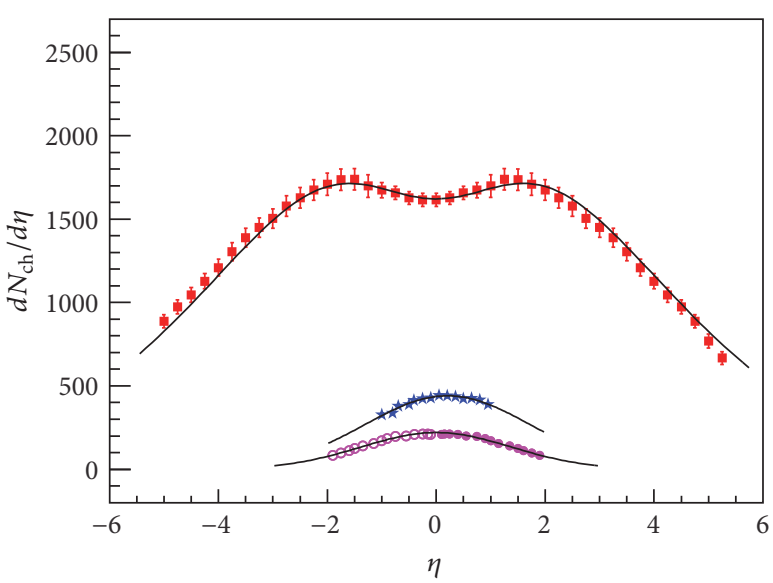

$$
\begin{aligned}
& \mathrm{Pb}+\mathrm{Pb}(0-5 \%) \\
& \text { ALICE } \\
& \text { + } 2.76 \mathrm{TeV} \\
& \mathrm{NA} 50 \\
& \text { * } 17.3 \mathrm{GeV} \\
& +8.76 \mathrm{GeV}
\end{aligned}
$$$$
\text { — Double Gaussian }
$$

FIGURE 16: The pseudorapidity distribution of charged particles in $\mathrm{Pb}+\mathrm{Pb}$ collisions at three different energies, fitted with double Gaussian function shown by solid lines.

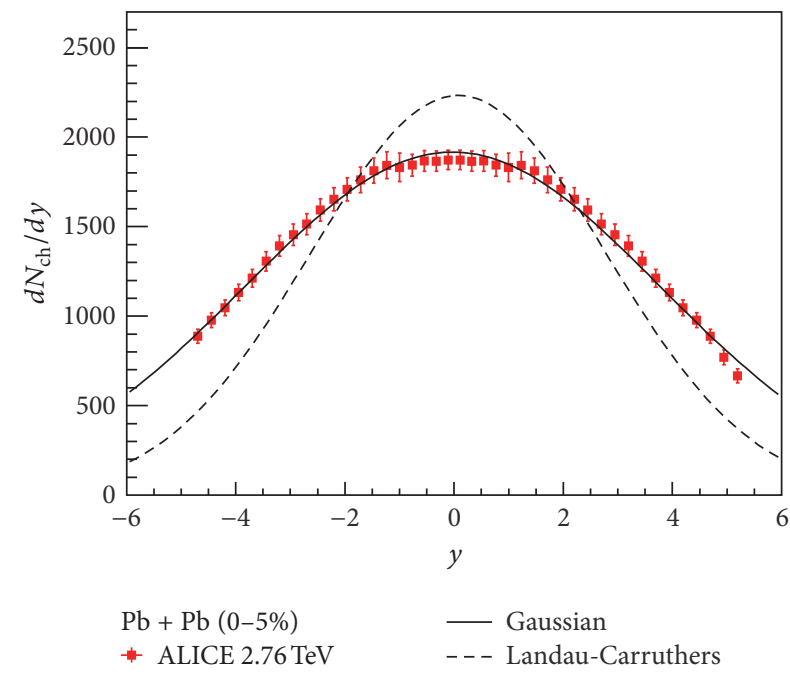

FIgURE 45: The charged particle rapidity distributions in $\mathrm{Pb}+\mathrm{Pb}$ collisions for the most central events at $\sqrt{s_{\mathrm{NN}}}=2.76 \mathrm{TeV}$ measured by ALICE experiment [2]. 


\section{References}

[1] R. Sahoo, A. N. Mishra, N. K. Behera, and B. K. Nandi, "Charged particle, photon multiplicity, and transverse energy production in high-energy heavy-ion collisions," Advances in High Energy Physics, vol. 2015, Article ID 612390, 30 pages, 2015.

[2] E. Abbas, B. Abelev, J. Adam et al., "Centrality dependence of the pseudorapidity density distribution for charged particles in $\mathrm{Pb}-\mathrm{Pb}$ collisions at $\sqrt{s_{N N}}=2.76 \mathrm{TeV}$,' Physics Letters $B$, vol. 726, no. $4-5$, pp. $610-622,2013$. 

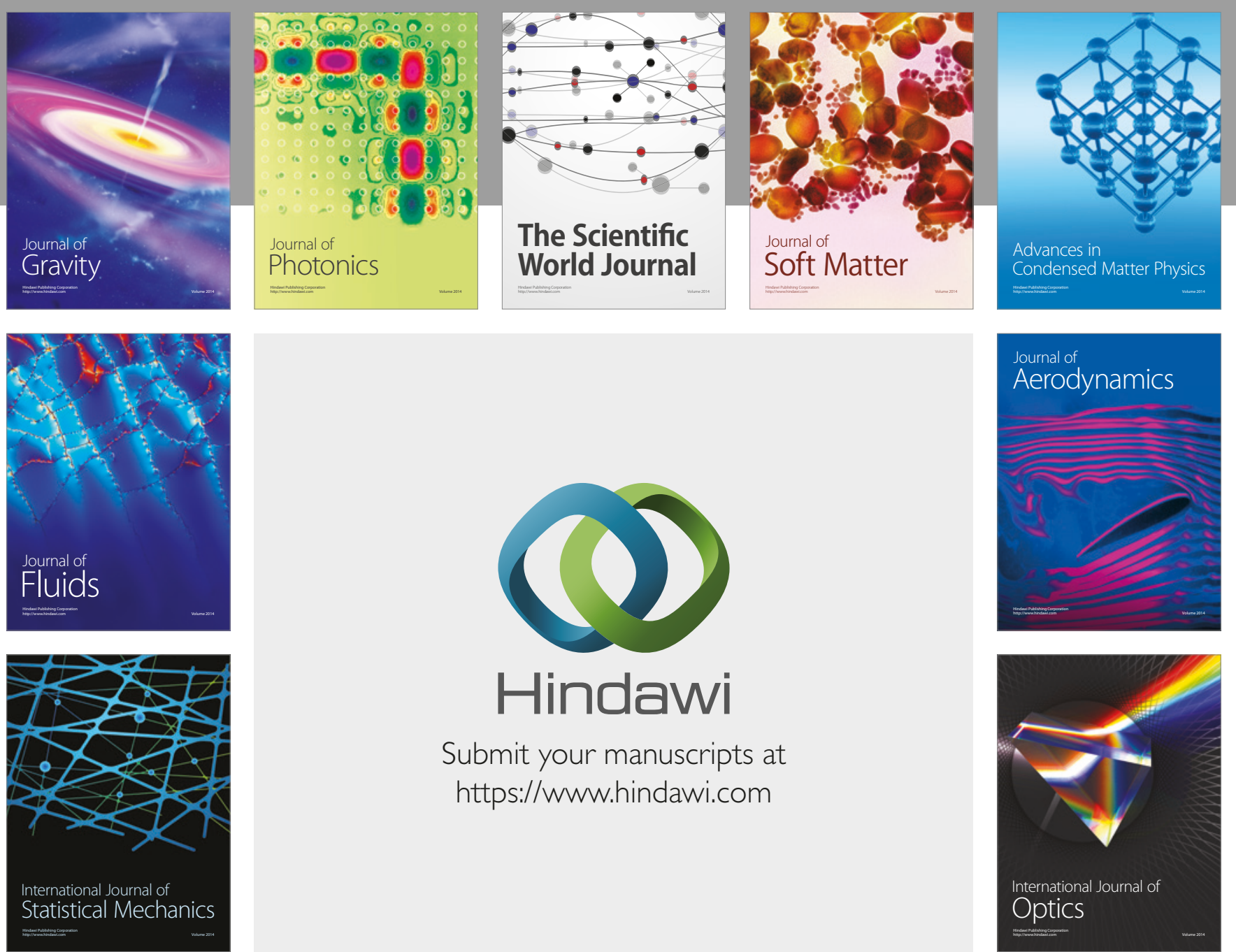

Submit your manuscripts at

https://www.hindawi.com
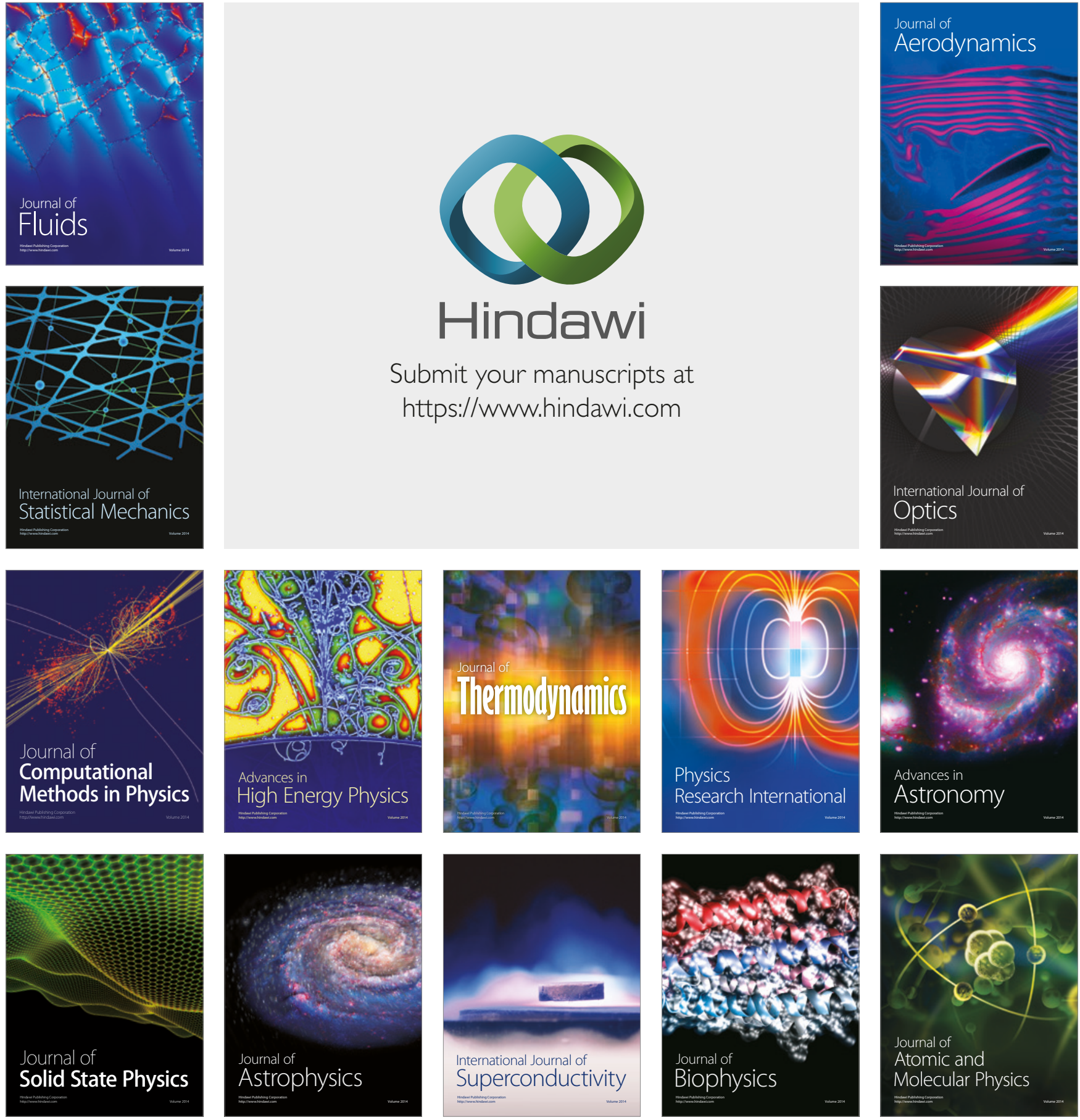